\title{
Anestesia para cirugía no cardíaca en pacientes pediátricos portadores de cardiopatías congénitas
}

\author{
$1^{\text {ra }}$ Parte
}

SILVANA CAVALLIERI B. ${ }^{1}$, FRANCISCO BOYE R. ${ }^{2}$

\section{Introducción}

a incidencia de Cardiopatías Congénitas (CC) es alrededor de 1 por 1.000 nacidos vivos. La patogenia se debe a factores genéticos y ambientales, aunque sólo en el $10 \%$ de los pacientes se logra identificar factores ambientales reconocibles.

Es indudable que hablar de CC es un tema muy amplio y que muchas veces escapa al conocimiento de un anestesiólogo pediátrico general. En el desarrollo de este artículo intentaremos dar respuestas a preguntas que se plantean con frecuencia ante un paciente con CC que debe someterse a una anestesia para cirugía no cardiaca. Es relevante comprender que estos pacientes, por su condición, tienen mayor morbilidad que pacientes de similar edad sin patología cardiaca agregada, por lo que un anestesiólogo general debe intentar comprender los aspectos básicos y esenciales de la anatomía y fisiología de la cardiopatía congénita que afecta al paciente. Durante la anestesia, debe plantearse un objetivo hemodinámico que sea favorable a la condición específica que determina la cardiopatía y poseer habilidades y conocimientos compatibles para el manejo anestésico de pacientes pediátricos para poder enfrentar las posibles complicaciones intraoperatorias.

\section{Desarrollo}

Las siguientes preguntas serán analizadas sucesivamente:

¿Por qué los anestesiólogos generales necesitamos saber de CC?

¿Cuáles con las CC más frecuentes con las que deberemos enfrentarnos?

¿Cómo orientarnos de manera fácil en la fisiopatología del paciente que enfrentamos?
¿Qué exámenes y que otros aspectos debemos tomar en cuenta en la evaluación preanestésica?

¿Cuáles son las condiciones de mayor riesco en pacientes con CC? ¿Existe una clasificación de riesgo? Una vez que conocemos su fisiopatología, ¿cómo debemos proceder para lograr el mejor equilibrio hemodinámico y general del paciente?

Cómo enfrentar cada una de las distintas cardiopatías, (las más frecuentes), en el caso de un paciente sometido a cirugía no cardiaca?

\section{¿Por qúe los anestesiólogos generales necesita- mos saber de CC?}

A medida que mejoran las condiciones socioeconómicas de un país, las enfermedades congénitas como las cardiopatías se van haciendo más relevantes, llegando a representar la enfermedad congénita más frecuente (30\%). Se estima una incidencia de $1 / 100$ nacidos vivos y en prematuros la incidencia es 3 veces más frecuente, con una alta mortalidad de no mediar intervención (15\% de sobrevida sin cirugía). La mortalidad quirúrgica global de las CC varía según el centro entre $5-10 \%$. Es por esto que la chance de que un anestesiólogo general se vea enfrentado a pacientes con este tipo de patología para cirugía no cardiaca es elevada. Como lo verán a lo largo de sus carreras profesionales, los pacientes no sólo llegan a intervenirse de una cirugía cardiaca, sino que, frecuentemente requieren otro(s) procedimiento(s). Indudablemente la condición cardiaca implica que son pacientes de mayor riesgo anestésico y muchas veces la CC está asociada a cromosomopatías como Síndrome de Down, Turner, di George, entre otras ${ }^{1}$ (50\%). Un problema emergente es la Cardiopatía Congénita en el Adulto (CCA). En Chile, también se está convirtiendo en un problema de salud pública,

Médico Anestesiólogo, Hospital Luis Calvo Mackenna, Clínica Las Condes.

2 Médico Cirujano Cardiovascular Pediátrico, Hospital Luis Calvo Mackenna, Clínica Santa María.

Correspondencia:

Silvana Cavallieri Badilla

E-mail: silvanacavallieri@gmail.com

Conflicto de interés declarado: ninguno. 
que sólo unos pocos centros del país están en condiciones de abordar.

En cuanto al riesgo anestésico, múltiples estudios han determinado que la patología cardiaca congénita se encuentra presente en un porcentaje alto de los niños que tuvieron paro cardiaco peri-operatorio². Dentro de las condiciones más complejas y que presumiblemente tendrás más eventos hemodinámicos desfavorables se encuentran los pacientes portadores de ventrículo único ${ }^{3}$, especialmente en la etapa I, los pacientes con obstrucción del tracto de salida ventricular y pacientes con cardiomiopatía. Las condiciones de menos riesgo son felizmente las más frecuentes y corresponden a los pacientes que presentan cortocircuito de izquierda a derecha. Si se considera la categoría (no reparada, reparada o paliada), los casos de paro cardiaco que se produjeron fueron en un $59 \%$ en pacientes con CC no reparada, en un $26 \%$ en paciente con procedimientos paliativos y en un $15 \%$ en pacientes con lesiones reparadas².

\section{¿Cuáles con las CC más frecuentes con las que deberemos enfrentarnos para cirugía no cardia- ca?}

En orden de frecuencia son las siguientes:

a. Comunicación interventricular.

b. Comunicación interauricular.

c. Ductus Arterioso Permeable.

d. Tetralogía de Fallot.

e. Coartación de la aorta.

f. Transposición de grandes arterias.

g. Ventrículo Único: Glenn, Fontan.

\section{¿Cómo orientarnos de manera fácil en la fisiopa- tología del paciente con CC?}

Existen numerosas clasificaciones. Muchas de ellas complejas y destinadas esencialmente para el especialista. Una aproximación simple consiste primero en definir si la cardiopatía es cianótica o no cianótica, luego si existe un shunt intra o extra cardiaco (y hacia qué dirección). Finalmente, si presenta flujo pulmonar aumentado o disminuido. Esta información se encuentra normalmente en la ecocardiografía más reciente tanto si el paciente aún no ha sido intervenido, como si se encuentra con la cardiopatía corregida o ha recibido una cirugía paliativa. Si existe una duda sobre la condición cardiológica es conveniente consultar al cardiólogo de cabecera ${ }^{3}$. A continuación se exhibe una clasificación operacional para el no especialista. (Tabla 1. Clasificación de las cardiopatías congénitas).

¿Qué aspectos generales debemos tomar en cuenta en la evaluación preanestésica, en la preparación preoperatoria y en postoperatorio?

Antecedentes: es fundamental tener una idea precisa de la historia cardiológica del paciente, de las intervenciones previas (correctoras o paliativas), la presencia de arritmias, si es usuario o no de marcapasos (y en qué modalidad), los medicamentos utilizados y las últimas dosis administradas.

Estado general: evaluar la capacidad funcional, el estado nutricional, la presencia de cicatrices, el estado del capital venoso. En forma dirigida se debe verificar el hematocrito actual (recordar que $<30 \%$ o $>$ $60 \%$ son límites considerados de riesgo), la saturación de oxígeno habitual y actual (saturación menor a $75 \%$ en un paciente con CC cianótica se considera de alto riesgo). En los exámenes de laboratorio debe ponerse atención al nivel de sodio y potasio plasmático, en particular si el paciente es usuario de diuréticos, está en ayuno prolongado o ha tenido episodios de vómitos o diarrea. Debe verificarse el estado de la dentadura en busca de sepsis dentaria por el riesgo de endocarditis bacteriana.

En relación con la cardiopatía debe revisarse la radiografía de tórax reciente si corresponde (silueta cardiaca -búsqueda de cardiomegalia-, signos de flujo pulmonar aumentado, presencia patología pulmonar agregada, entre otras). En el electrocardiograma la presencia de arritmias. Revisar el ecocardiograma más

\begin{tabular}{|c|c|}
\hline No Cianoticias & Cianoticias \\
\hline Con Shunt de Izquierda a Derecha & Con flujo pulmonar disminuido \\
\hline $\begin{array}{l}\text { - Comunicación interventricular, Comunicación interauri- } \\
\text { cular, ductus arterioso persistente, canal auriculoventricular }\end{array}$ & - Tetralogía de Fallot \\
\hline Con obstrucción al flujo sistémico & Con flujo pulmonar normal o aumentado \\
\hline $\begin{array}{l}\text { - Coartación aortica, estenosis aortica, miocardiopatía hiper- } \\
\text { trófica }\end{array}$ & - Ventrículo de doble salida \\
\hline Miocardiopatías & \\
\hline
\end{tabular}


reciente y los previos (si existiesen) haciendo énfasis en la anatomía actual, función valvular, función ventricular (sistólica y diastólica) y presencia de stents.

Por último, la visita pre anestésica es el momento de informar a los padres del plan anestésico, de los riesgos y del destino postoperatorio más probable del paciente.

Para planificar la anestesia de un paciente cardiópata en la edad pediátrica se requiere un "Ambiente pediátrico", es decir disponer de los elementos, dispositivos, equipo y ayuda apropiada.

Con respecto al equipamiento este debe ser el estándar de un pabellón pediátrico, al cual se debe agregar la disponibilidad de un desfibrilador con palas apropiadas al tamaño del paciente, sistemas para mantener la temperatura, sistemas de administración cuidadosa de líquidos (bombas de infusión y sistemas de perfusión pediátricos.

La planificación de la anestesia debe realizarse tomando en cuenta la cardiopatía, el grado de impacto en la función cardiaca y el impacto de la patología no cardiaca actual que amerita la cirugía. Es ideal disponer de un acceso venoso antes de la inducción anestésica, en el caso de no tenerlo, realizar siempre una inducción inhalatoria cuidadosa y lenta, con drogas que idealmente no depriman la contractilidad (excepto en casos muy específicos). Debe instalarse siempre la monitorización estándar no invasiva, evaluar adecuadamente la necesidad de instalar monitoreo invasivo, sobre todo cuando se avizora la medición frecuente del estado metabólico y ácido base. Es esencial manejar el paciente neonatal con extremo cuidado, considerando que el miocardio neonatal tiene una curva de respuesta 4 , (ley de Frank-Starling), mucho más aplanada que un niño mayor y por lo tanto, está mucho menos preparado para tolerar aumentos bruscos de la pre y la post carga. La contractilidad del miocardio neonatal es además muy dependiente de la concentración de calcio extra e intracelular, por lo que este médicamente debe encontrarse en el arsenal disponible para ser utilizado en diluciones adecuadas en caso de necesitarlo.

En términos generales en los pacientes cardiópatas se debe evitar la administración inadvertida de burbujas por las líneas venosas o arteriales y realizar la profilaxis antibiótica de acuerdo a las normativas de cada centro, las que en general se ajustan a las últimas recomendaciones para la prevención de endocarditis infeccios $a^{5}$. Actualmente las indicaciones de profilaxis antibiótica de endocarditis infecciosa son más restringidas que en el pasado. En cualquier caso hay que tener en cuenta que el paciente puede tener una indicación de profilaxis antibiótica por la con- dición cardiaca, pero también por la condición quirúrgica. Muchos pacientes están con medicamentos que conviene mantener durante el período peri operatorio. Típico ejemplo son los pacientes con shunt de izquierda a derecha que suelen estar bajo terapia diurética para disminuir los síntomas de insuficiencia cardiaca, los pacientes portadores de ventrículo único que frecuentemente están con inhibidores de la enzima convertidora renina-angiotensina y los pacientes con Tetralogía de Fallot que suelen estar bajo terapia con B bloqueadores. Algunos pacientes también pueden estar con anti-agregantes plaquetarios (aspirina) o con inhibidores de la vitamina K. Los anticoagulantes se deben suspender transitoriamente para cirugías invasivas, pero no para procedimientos menores sin riesgo de sangrado.

Con respecto al manejo postoperatorio de un paciente con CC dependerá de la magnitud del defecto y de la cirugía a la que será sometido. Defectos pequeños, sin gran repercusión y cirugías simples no ameritan un cuidado especial en el postoperatorio. Si se trata de defectos relevantes, con repercusión anatómica y/o fisiopatológica, paciente con factores de riesgo y/o de una cirugía mayor, debe motivar una discusión del anestesiólogo con el cardiólogo y el cirujano tratante sobre el destino postoperatorio más adecuado.

\section{¿Existe una clasificación de riesgo de las cardio- patías congénitas?. ¿Cuáles son las condiciones de mayor riesgo en pacientes con CC?}

Las cardiopatías congénitas están consideradas en la clase III (ASA III) de la American Society Of Anesthesia. Sin duda existen algunas que son más graves que otras. Se puede considerar que los pacientes que presentan las siguientes condiciones son más graves y el anestesiólogo debe estar alerta ante su presencia:

- Hipoxemia crónica: $\mathrm{SaO}_{2}<75 \%$.

- Hematocrito > 60\%.

- Flujo pulmonar vs Sistémico > 2:1.

- Obstrucción flujo sistémico o pulmonar > 50 $\mathrm{mmHg}$.

- Hipertensión pulmonar.

- Insuficiencia cardiaca.

- Arritmias.

- Marcapasos.

- Uso medicamentos (diuréticos, anticoagulantes, antiarrítmicos, vasodilatadores).

- Enfermedades asociadas.

Una vez que conocemos la fisiopatología ¿cómo debemos proceder para lograr el mejor equilibrio hemodinámico y general del paciente?

Antes de pasar a revisar cada una de las patolo- 
gías, se revisará brevemente cuáles son los parámetros fisiológicos que pueden manipularse durante la anestesia y en qué sentido podemos hacerlos variar. Este enfrentamiento será útil para que después al analizar cada patología el lector pueda decidir qué objetivos hemodinámico debe plantearse y como conseguirlos. (Tabla 2. Grilla de parámetros que se pueden manipular durante la anestesia).

Al momento de enfrentar una determinada cardiopatía también es relevante tomar en cuenta cómo las drogas anestésicas afectaran la función cardiovascular. (Tabla 3. Efectos de distintos fármacos sobre los parámetros hemodinámicos).

Los anestésicos inhalatorios ${ }^{6}$ deprimen la contractilidad fundamentalmente por la disminución del calcio disponible para el miocardio, afectando el flujo de calcio al retículo sarcoplásmico, con un efecto neto de disminuir los depósitos de calcio intracelular. El halotano disminuye más que el isoflurano la contractilidad, probablemente porque reduce más acentuadamente el peak intracelular de calcio durante la contracción. Los estudios en humanos o animales de corta edad varían en sus resultados, dependiendo además de la edad de los sujetos (fetal, neonatal, lactante, niño mayor). Todos los anestésico inhalatorios, tienen la capacidad de disminuir la contractilidad cardiaca, pero este efecto es particularmente relevante con halotano. Es probable que la baja disponibilidad de calcio en el sarcolema sea en parte responsable de la depresión miocárdica dosis dependiente, particularmente en el neonato. Este efecto se prolonga hasta las 6-12 semanas de edad postnatal. Con respecto a los anestésicos halogenados más nuevos; el sevoflurano parece tener el mejor perfil hemodinámico para

\begin{tabular}{ll}
\multicolumn{2}{c}{ Tabla 2. Grilla de parámetros que se pueden manipular durante la anestesia } \\
\hline Parámetro & Efecto buscado \\
Contractilidad & Mantener, disminuir o aumentar \\
Frecuencia cardiaca & Mantener, disminuir o aumentar \\
Precarga & Mantener, disminuir o aumentar \\
Resistencia vascular sistémica & Mantener, disminuir o aumentar \\
Resistencia vascular pulmonar & Mantener, disminuir o aumentar \\
\hline
\end{tabular}

\begin{tabular}{|c|c|c|}
\hline \multirow[t]{2}{*}{ Contractilidad } & Aumentan $\uparrow$ & Disminuyen $\downarrow$ \\
\hline & $\begin{array}{l}\text { - Calcio } \\
\text { - Dopamina, Dobutamina, Adrenalina, } \\
\text { Noradrenalina } \\
\text { - Inhibidores de la Fosfodiesterasa }\end{array}$ & $\begin{array}{l}\text { - Halogenados (halotano) } \\
\text { - Anticálcicos (transfusiones) }\end{array}$ \\
\hline Frecuencia cardiaca & $\begin{array}{l}\text { - Atropina } \\
\text { - } \beta \text {-estimulantes } \\
\text { - Isoflurano, sevoflurano (en MAC ha- } \\
\text { bitual }\end{array}$ & - Opioides: fentanilo, remiferntanilo \\
\hline Precarga & $\begin{array}{l}\text { - Volumen } \\
\text { - Vasconstrictores } \rightarrow \text { fenilefrina } \\
\text { - Maniobras como uso de PEEP, Com- } \\
\text { presión abdominal }\end{array}$ & $\begin{array}{l}\text { - Pérdidas de volumen (sangrado, dia- } \\
\text { rrea, ayuno) } \\
\text { - Uso diuréticos } \\
\text { - Nitritos (nitroglicerina, nitroprusiato) }\end{array}$ \\
\hline Resistencia sistémica & $\begin{array}{l}\text { - Ketamina } \\
\text { - Vasconstrictores } \rightarrow \text { alfa agonistas, fe- } \\
\text { nilefrina, dopamina en dosis altas } \\
\text { - Compresión abdominal }\end{array}$ & $\begin{array}{l}\text { - Isoflurano, propofol } \\
\text { - Inhib fosfodiesterasa } \\
\text { - Nitritos (nitroglicerina, nitroprusiato) }\end{array}$ \\
\hline Resistencia Pulmonar & $\begin{array}{l}\text { - Hipoxia } \\
\text { - Hipercarbia } \\
\text { - PEEP } \\
\text { - Acidosis }\end{array}$ & $\begin{array}{l}\text { - Hiperoxia, hipocarbia, alcalosis } \\
\text { - Inhib fosfodiesterasa } \\
\text { - Óxido nítrico, PGE1 } \\
\text { - Hematocrito bajo }\end{array}$ \\
\hline
\end{tabular}


utilizar en niños a cualquier edad, ya que además se agrega su buena tolerancia a la inducción inhalatoria, lo que no ocurre ni con isoflurano ni con desflurano que, teniendo un perfil de impacto hemodinámico relativamente bueno a $1 \mathrm{MAC}$, son pungentes para la inducción inhalatoria y por lo tanto, deben descartarse de plano en un niño cardiópata durante la inducción. Como el isoflurano, el desflurano produce vasodilatación, lo que da lugar a reducciones dependientes de la dosis en la resistencia vascular sistémica y la presión arterial, pero si se requiere elegir un anestésico para manejar la resistencia periférica, es probable que el isoflurano tenga preeminencia sobre el desflurano, ya que existe mayor experiencia en niños con el primero. El isoflurano no tiene efectos irritativos sobre el árbol bronquial (el desflurano produce tos, laringo y broncoespasmo e hipersecreción bronquial).

El efecto de los distintos anestésicos inhalatorios sobre el sistema cardiovascular se puede apreciar en la tabla siguiente ${ }^{7}$ (Tabla 4. Efecto de los anestésicos inhalatorios sobre el sistema cardiovascular).

El Propofol ${ }^{3,7}$ es un fármaco de amplio uso actualmente en pediatría, sin embargo, su uso en cardiopatías congénitas debe ser muy cuidadoso ya que provoca una caída significativa de la Resistencia Vascular Sistémica (RVS) y de la presión arterial media, por lo que se recomienda no usar, o ser cuidadosos, en pacientes con shunt de Derecha a Izquierda, (situación en la que se depende de la presión arterial sistémica para mantener un flujo pulmonar apropiado) y en pacientes con debito sistémico ya comprometido. El gráfico siguiente muestra el efecto muy relevante que tiene la administración de $2 \mathrm{mg} / \mathrm{kg}$ de propofol en pacientes portadores de una cardiopatía congénita. En los recién nacidos sin cardiopatía el propofol también produce una caída importante y sostenida de la presión arterial. En estos pacientes la Resistencia Vascular Sistémica presenta una caída promedio del 20\% de los valores basales ${ }^{8}$. (Figura 1. Efecto hemodinámico de propofol administrado a niños con cardiopatía congénita).
Con respecto a la Ketamina7, esta droga actúa a través de la inhibición de receptor N-Metil-D-Aspartato (NMDA) en el cerebro y por lo tanto, con un mecanismo distinto a los anestésicos inhalatorios. Aunque es un depresor miocárdico directo, luego de su administración se produce un aumento de la frecuencia cardiaca, de la presión arterial y del débito cardiaco debido a la estimulación del sistema simpático, con aumento de las catecolaminas circulantes, además con una inhibición de la recaptación de catecolaminas. La Ketamina puede usarse por vía intravenosa (1-2 mg/kg) o intramuscular (5-7 mg/kg). Debido a sus mínimos efectos sobre la RVS, Presión Arterial Media (PAM), Resistencia Vascular Pulmonar (RVP) y Presión Arterial (PA), es útil en pacientes con buena función cardiaca, o cuando la función esta moderadamente comprometida. La Ketamina también es muy utilizada en pacientes portadores de cardiopatía congénita, especialmente en neonatos y en pacientes cianóticos, en pacientes con insuficiencia cardiaca, con hipertensión pulmonar (no aumenta la presión pulmonar de manera relevante). Hay que usarla con mucha precaución en pacientes que se encuentra estimulados al máximo con $\beta$ adrenérgicos ya que en ellos, los efectos depresores miocárdicos de la Keta-

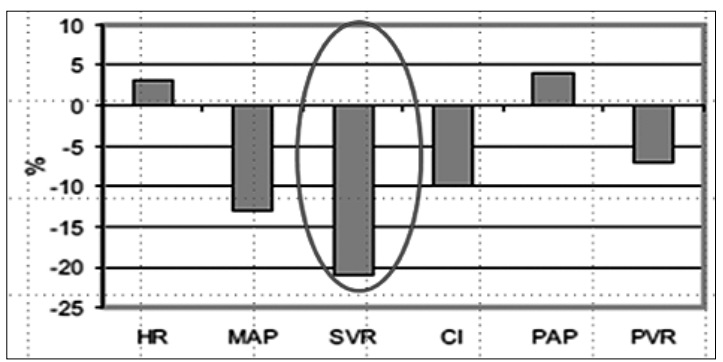

Figura 1. Efecto hemodinámico de Propofol administrado a niños con cardiopatía congénita. La resistencia vascular sistémica presenta una caída que en promedio alcanza el 20\% de los valores basales. Adaptado de Seminars in Cardiothoracic and Vascular Anesthesia. 2014, 18(4); 363 - 70.

Tabla 4. Efecto de los anestésicos inhalatorios sobre el sistema cardiovascular

\begin{tabular}{lccccc}
\hline & N2O & Halotano & Isoflurano & Sevoflurano & Desflurano \\
Frecuencia cardiaca & $\leftrightarrow$ & $\downarrow$ & $\uparrow$ & $\uparrow$ Inducción & $\uparrow$ \\
Presión arterial sistémica & $\leftrightarrow$ & $\downarrow$ & $\downarrow$ & $\downarrow$ & $\downarrow$ \\
Inotropismo & $\downarrow$ & $\downarrow \downarrow$ & - & $\downarrow$ & $\downarrow$ \\
Débito cardiaco & $\leftrightarrow$ & - & $\downarrow \downarrow$ & $\downarrow$ & - \\
RVS & - & - & Leves & Leves & Leves \\
Arritmias & - & Importantes & & $\downarrow$ & $\downarrow$ \\
\hline
\end{tabular}


mina no podrán ser contrarrestados por un aumento de las catecolaminas, (que es el efecto que se produciría en condiciones normales) y puede desencadenarse una caída dramática del débito cardiaco. En pacientes con falla cardiaca extrema es mejor utilizar solamente opioides y dosis bajas de benzodiacepinas si es necesario.

La dexmedetomidina 7,8 , un $\alpha_{2}$ agonista que actúa a nivel post-sináptico en la médula espinal, produciendo analgesia discreta y en el cerebro produciendo sedación, se está utilizando cada vez más en unidades de cuidado intensivos y en estudios hemodinámicos en pacientes cardiópatas. Sin embargo, su uso durante la anestesia para cirugía no cardiaca, en manos de no especialistas no está suficientemente estudiado.

\section{¿Cómo enfrentar cada una de las distintas car- diopatías (las más frecuentes) en el caso de un paciente sometido a cirugía no cardiaca?}

A continuación se analizará sucesivamente el manejo específico de cada patología entre las más frecuentes a las que puede verse enfrentado un anestesiólogo general para cirugía no cardiaca.

\section{Comunicación interventricular}

Ocurre entre 1,5 a 3,5 por mil nacidos vivos representando el $20 \%$ de todas las CC. Un defecto septal ventricular o comunicación interventricular (CIV) es una comunicación anormal entre los dos ventrículos, y es la cardiopatía congénita más común. Puede haber un componente genético y ambiental. La etiología no es bien conocida. Las CIV ocurren con frecuencia como defectos aislados, pero también pueden ser un componente más de otras anomalías congénitas, como la Trisomía 13, 18 y 21, de la malformación llamada asociación VACTERL (síndrome poli-malformativo que se caracteriza por anomalía Vertebral, Ano imperforado, anomalía Cardiaca, fístula TraqueoEsofágica, anomalías Renales y de extremidades) y del síndrome de CHARGE (Coloboma, defectos cardiacos, Atresia de coana, Retardo crecimiento/psicomotor, alteraciones Genitourinarias, anomalías del oído/ sordera).

Las CIV se clasifican generalmente de acuerdo a su localización en el septum interventricular, en cinco tipos:

1. Perimembranosa: (membranosa, paramembranosa, subaórtica, infracristal) es el tipo más frecuente. Envuelven el septo membranoso, bordeado por la válvula Aurículo-Ventricular (AV) derecha. Mu- chas veces la válvula tricúspide está comprometida por tejido fibroso de su aparato subvalvular que tiende a fijar los velos valvulares hasta incluso poder ocluir el defecto. Anomalías de la comisura de la válvula aórtica pueden coexistir. Este defecto pueden estar presente con mal alineamiento del tabique interventricular, típicamente como parte de una cardiopatía congénita más compleja.

2. Cono ventricular: (subaórtica, conal, conoseptal, conoventricular, doblemente relacionada, infundibular, infracristal): se encuentran entre el septum conal y muscular. Está frecuentemente asociada a algún grado de mal alineamiento de los bordes del septum interventricular que la forman. Por ejemplo: mal alineamiento anterior en la Tetralogía de Fallot, mal alineamiento posterior en la interrupción del arco de la aorta. A diferencia de la CIV peri-membranosa, ésta no tiene borde membranoso y frecuentemente es no-restrictiva.

3. Subpulmonar: (sub-pulmonar, supra-cristal o de salida) 5 a 7\% de los defectos; Situado debajo de la válvula pulmonar, en el septo de salida. El resultado es el desarrollo de un flujo de salida desde el ventrículo izquierdo hacia la arteria pulmonar. Los velos valvulares aórticos podrían prolapsarse a través del defecto, dando como resultado el cierre parcial del defecto o insuficiencia aórtica.

4. Inlet: (también Ilamada tipo canal atrio-ventricular $(\mathrm{AV})$, septum AV o posterior), representan el 5 a $10 \%$ de las CIV. Ubicado en la zona del tabique formado por tejido de cojinete endocárdico, inmediatamente inferior a la válvula tricúspide. Está asociado con anomalías de las Válvulas AV, considerada parte de los defectos del tabique auriculoventricular (AVSD).

5. Muscular: (se pueden subdividir según la ubicación, en anterior, media, apical, marginal o posterior) representan 5 a $20 \%$ de defectos; el defecto está completamente rodeado por músculo. Habitualmente se relacionan en la zona donde la banda moderadora emerge desde la banda septal del ventrículo derecho. El defecto puede ser único o pueden ser múltiples.

También se pueden clasificar por tamaño, en relación con el tamaño del anillo aórtico:

- Pequeño: menos de 1/3 del diámetro normal del anillo de la válvula aórtica.

- Moderado: 1/3 a 2/3 del diámetro del anillo de la válvula aórtica.

- Grande: más de 2/3 del tamaño normal del anillo de la válvula aórtica. 


\section{Fisiopatología}

Clínicamente los síntomas de un paciente portador de una CIV son variables de acuerdo al tamaño del defecto. Las CIV hemodinámicamente significativas se presentan con signos de insuficiencia cardiaca (taquipnea, alteración del desarrollo pondo-estatural, desnutrición, anemia). A la auscultación se evidencia un soplo pansistólico.

Las consecuencias fisiopatológicas se manifiestan por:

- Shunt de izquierda a derecha a través del defecto. Dependiendo de la RVP y de la presión pulmonar, éste puede cambiar o combinar la dirección del flujo (bidireccional o de derecha a izquierda).

- Insuficiencia cardiaca congestiva.

- Hipertensión pulmonar.

Al existir un defecto en el tabique que separa ambos ventrículos hay paso de sangre desde el ventrículo izquierdo al ventrículo derecho y al tronco de la arteria pulmonar (TAP). Como el shunt se produce en el sístole ventricular, las cavidades sobrecargadas son las izquierdas (aurícula y ventrículo izquierdo) con aumento de flujo a los pulmones. Las cavidades izquierdas con el tiempo se dilatan, lo que sumado a congestión pulmonar determinará que frecuentemente el paciente presente sintomatología respiratoria.

La sintomatología dependerá de la mezcla de:

- Tamaño del defecto: una CIV se clasificará según su relación al tamaño del anillo de la aorta. Menor al $50 \%$ de éste se denominará restrictiva y sobre $50 \%$ es no-restrictiva. Cabe hacer notar que también entran en juego la posición de la CIV, la RVP (recordar que ésta cae progresivamente en los primeros 4 meses de vida) y el tamaño del paciente.

- RVS.

- RVP.

- Asociación con otros defectos intracardiacos.

Funcionalmente, la repercusión de la CIV se evaluará mediante la relación entre el flujo pulmonar (Qp) y el flujo sistémico (Qs). Se expresará en un cociente: Qp:Qs.

- Mínima: Qp:Qs inferior a 1,5.

- Moderado: Qp:Qs entre 1,5 a 2,0.

- Elevado: Qp:Qs superior a 2,0.

Como se dijo anteriormente, los síntomas varían de acuerdo al tamaño del defecto, y también de acuerdo a otras condiciones como existencia de otro defecto asociado, presencia de anemia, grado de resistencia vascular pulmonar. En general pueden distinguirse los siguientes cuadros:
- Defecto pequeño. Limita el cortocircuito (shunt): Mínima sobrecarga de volumen del ventrículo izquierdo, RVP normal a levemente elevada.

- Defecto moderado. Hay moderado cortocircuito de izquierda a derecha, se presenta sobrecarga de volumen del ventrículo izquierdo con dilatación de las cavidades izquierdas e hipertrofia del ventrículo izquierdo, la resistencia vascular pulmonar moderadamente elevada y el tamaño del ventrículo derecho es normal.

- Defecto grande (no hay limitación al cortocircuito de Izquierda a Derecha). La resistencia al flujo está determinada por la relación de la RVP comparada con la resistencia vascular sistémica. Hay sobrecarga de volumen y de presión en la vasculatura pulmonar (y de vuelta a las cavidades izquierdas). Sumado a la dilatación de las cavidades izquierdas, puede haber hipertrofia y dilatación del ventrículo derecho dado que las RVP y presión pulmonar están elevadas.

Los pacientes que presentan defectos grandes, si no son tratados desarrollarán gradualmente hipertensión pulmonar. Potencialmente se podría desarrollar enfermedad vascular pulmonar irreversible, conocido como Síndrome de Eisenmenger. Esto implica que las presiones en el territorio pulmonar son superiores a la presión sistémica, con lo cual se revierte el flujo y pasa a ser un shunt de derecha a izquierda. El paciente se volverá progresivamente cianótico. Al día de hoy esto marca un cuadro prácticamente irreversible. En raras ocasiones, requiere medicación especial para tratar de disminuir la presión pulmonar. La alternativa quirúrgica de cerrar la CIV en estos casos se vuelve de un resultado incierto.

\section{Evaluación preanestésica}

Es relevante conocer la historia de la cardiopatía, determinar el impacto fisiológico, hospitalizaciones y cirugías previas.

En el examen físico, y dependiendo del tamaño del defecto, el paciente puede tener un grado variable de disnea, congestión pulmonar, taquicardia y anemia. Debe evaluarse la presencia de complicaciones pulmonares como Neumonía Secundaria a la congestión pulmonar (que puede requerir optimización antes de una cirugía electiva). Cuando el defecto está asociado a otros síndromes genéticos habrá que hacer una evaluación específica de los sistemas afectados por el síndrome.

Se debe inquirir sobre la medicación, estos pa- 
cientes habitualmente se encuentran bajo tratamiento con diuréticos y vasodilatadores, por lo que pueden tener algunas alteraciones metabólicas como alcalosis hipokalémica. (Figura 2. Comunicación Interventricular: Shunt de izquierda a derecha a nivel de ventrículos).

\section{Anestesia}

Para minimizar el aumento del shunt mientras el paciente está anestesiado, se busca aumentar la RVP y disminuir la RVS, tratando de llevar al paciente a un Qp:Qs lo más cercano a 1:1. Para ello, el anestesiólogo debe ventilar con las concentraciones de oxígeno lo más bajas posibles, idealmente con $\mathrm{F}_{\mathrm{i}} \mathrm{O}_{2}$ de $21 \%$, además hipoventilar para lograr una hipercapnia con niveles de $\mathrm{pCO}_{2}$ sobre $40 \mathrm{mmHg}$ (hasta $50 \mathrm{mmHg}$ ) y uso de ventilación positiva al final de la espiración (PEEP). Recordar que la hiperventilación no es conveniente para estos pacientes, tanto porque aumenta el flujo pulmonar como porque acentúa la alcalosis y la hipokalemia. (Tabla 5. Grilla para el manejo hemodinámico durante la anestesia en un paciente portador de CIV).

Respecto de los anestésicos a utilizar; debe evitarse disminuir la contractilidad para no precipitar una falla cardiaca. En caso de inducción inhalatoria, el sevoflurano es una droga apropiada pero debe ser usada en forma cuidadosa, la mantención puede realizarse con sevoflurano o isoflurano, dependiendo de si el paciente será extubado al final de la cirugía, en cuyo caso el perfil de sevoflurano es más apropiado, aunque hay que prever que se puede presentar agitación post-anestésica en un porcentaje de los pacientes. Si se presenta esta complicación una dosis baja de Propofol (1 a $2 \mathrm{mg} / \mathrm{Kg}$ ) administrada lentamente durante la emergencia anestésica, permite manejar el síntoma.

Cuando la inducción es por vía intravenosa puede

\begin{tabular}{|c|c|}
\hline \multicolumn{2}{|c|}{$\begin{array}{l}\text { Grilla para el manejo hemodinámico durante la } \\
\text { anestesia en paciente portador de CIV }\end{array}$} \\
\hline Contractilidad & $=0 \downarrow$ \\
\hline Función cardiaca & $=$ \\
\hline Precarga & $\uparrow$ \\
\hline RVP & $\uparrow$ \\
\hline RVS & $\downarrow$ \\
\hline
\end{tabular}

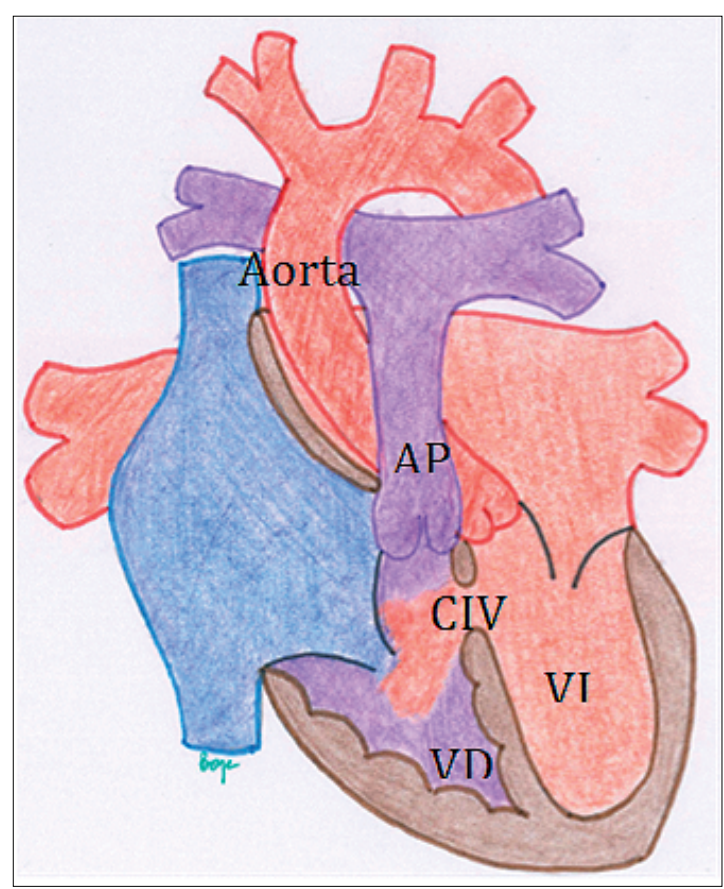

Figura 2. Comunicación interventricular: Shunt de izquierda a derecha a nivel de los ventrículos. Este se produce en sístole y en dirección a la arteria pulmonar con sobrecarga en las cavidades izquierdas. El Qp:Qs está aumentado.

utilizarse Propofol ya que provoca una caída de la RVS Esta caída de la RVS es un efecto deseado en las CIV, (porque disminuye el shunt de izquierda a derecha). Debe tenerse en cuenta que en pacientes pequeños, con función cardiaca límite, la caída de la RVS puede también generar una caída del débito por mala perfusión miocárdica, por lo tanto, la utilización de Propofol debe ser muy cuidadosa en este tipo de pacientes. La Ketamina en general no provoca cambios hemodinámicos relevantes. El uso de opioides es beneficioso en pacientes con cortocircuito de izquierda derecha porque disminuyen moderadamente la resistencia vascular sistémica disminuyendo la relación Qp:Qs.

Cualquier relajante muscular es apropiado, adaptando la dosis al tipo y longitud esperada de la cirugía. Si el paciente se encuentra en estado crítico y se espera un postoperatorio en ventilación mecánica, el uso de opioides y benzodiacepinas asociado o no con concentraciones bajas de halogenados es apropiado. En este caso puede ser necesario usar inótropos, de los cuales la dopamina es la droga vasoactiva más utilizada habitualmente. La dobutamina en teoría no debería utilizarse dada su acción sobre la vasculatura pulmonar (vasodilatación). 
Respecto del manejo ventilatorio, es apropiado utilizar concentraciones bajas de oxígeno y mantener la $\mathrm{pCO}_{2}$ en cifras normales a moderadamente elevadas. Pero también es necesario considerar que en los pacientes con cortocircuito importante de izquierda a derecha puede existir congestión pulmonar que disminuirá la reserva respiratoria y provocará hipoxemia por breves que sean los períodos de pérdida de la vía aérea y de la ventilación.

Cabe mencionar que frecuentemente los pacientes con CIV no restrictiva y hemodinámicamente significativa, no tiene saturaciones de $\mathrm{O}_{2}$ cercanas a $100 \%$, sino más bien se presentan con saturaciones entre $75 \%$ a $85 \%$. Esta condición no significa que el paciente esté cursando con un síndrome de Eisenmenger, sino que el paciente presenta una disminución de la superficie de intercambio pulmonar por congestión pulmonar (más frecuentemente visto en los pacientes con síndrome de Down). En este caso es muy importante no hiperventilar para lograr saturaciones normales, ya que iatrogénicamente podría aumentar el Qp:Qs.

La monitorización dependerá de la condición del paciente como del tipo de cirugía. Si se trata de una cirugía de urgencia (cuadros sépticos con compromiso hemodinámico), es apropiado instalar una línea arterial y un catéter venoso central para la administración de medicamentos y drogas vasoactivas tanto en el intra- como en el postoperatorio. Si el paciente se encuentra en condición crítica debe evitarse la anemia (mantener hematocrito sobre 30\%, ya que esa condición acentuará la insuficiencia cardiaca (disminución de la viscosidad sanguínea). En los pacientes neonatos, debido a la presencia de una RVP elevada, el shunt a través del defecto es menor y no requieren de un manejo diferente al de recién nacido sin cardiopatía, nuevamente se debe evitar el disminuir la RVP, lo que podría llevar a que el paciente desarrolle insuficiencia cardiaca en el intra y postoperatorio.

\section{Comunicación interatrial}

Es un defecto en el tabique que divide ambos atrios. Las comunicaciones inter-atriales (CIA) se clasifican en los siguientes tipos, dependiendo de la ubicación en el septum:

- Ostium secundum (85\%).

- Ostium primium (10\%).

- Seno venoso (5\%).

- Seno coronario.

En casi todos los pacientes con defectos pequeños (menores a $3 \mathrm{~mm}$ de diámetro) ocurre un cierre espontáneo alrededor del año y medio de vida, también se cierran espontáneamente la mayoría de los defectos entre 3 - $8 \mathrm{~mm}$. Los defectos mayores requieren de un cierre ya sea percutáneo o quirúrgico.

Aunque no es una cardiopatía congénita, también se debe mencionar el Foramen Oval Permeable (FOP), que corresponde a una válvula cardiaca. El FOP está abierto en la vida intrauterina, permitiendo el paso de sangre oxigenada que viene de la vena cava inferior (previamente del ductus venoso, cordón umbilical y placenta) hacia el atrio izquierdo. Esto favorece que la sangre más oxigenada sea distribuida al cerebro del feto. La relevancia de este está que en algunos pacientes podría producirse una embolía paradojal en relación a aumento de presión intratorácica (maniobra de Valsalva o accesos de tos) y la administración inadvertida de burbujas por líneas de acceso venoso.

\section{Fisiopatología}

En general son pacientes asintomáticos y el defecto se cierra espontáneamente alrededor del año y medio de vida. En los defectos más grandes hay un cortocircuito importante de izquierda a derecha y a diferencia de la comunicación interventricular, (en la cual hay sobrecarga de volumen de las cavidades izquierdas), aquí se produce sobrecarga de volumen de las cavidades derechas. El shunt se produce en el diástole ventricular y si el defecto es muy amplio fisiológicamente no hay una gradiente de presiones. Dado que la complacencia del VD es mayor que la del VI, el primero es capaz de admitir más sangre. (Figura 3. Comunicación interatrial: Shunt de izquierda a derecha a nivel de atrios).

En cualquier paciente en que se encuentre cardiomegalia a expensas del ventrículo derecho en la radiografía de tórax es mandatorio descartar la presencia de una comunicación interauricular. Habitualmente la CIA es asintomática, pero en el caso que sea significativa, se presenta con disnea, letargia, y en general con escasa sintomatología de insuficiencia cardiaca. Si el paciente con un defecto relevante se deja evolucionar sin tratamiento, se pueden producir arritmias y más tardíamente desarrollarse un Síndrome de Eisenmenger, tal como se describió para la Comunicación Interventricular.

\section{Anestesia: Evaluación preanestésica}

En la mayor parte de estos pacientes el examen 


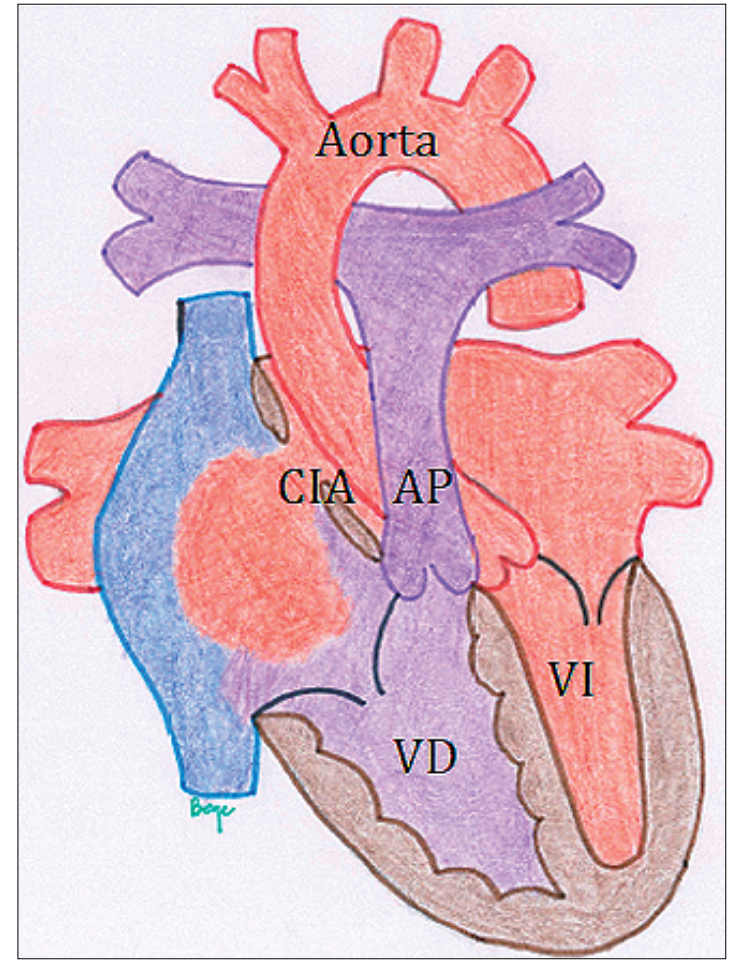

Figura 3. Comunicación interatrial: Shunt de izquierda a derecha a nivel de atrios. Éste se produce en el diástole y en dirección a las cámaras derechas debido a la mayor compliance de éstas. El flujo pulmonar está aumentado.

físico es anodino, puede auscultarse desdoblamiento persistente del segundo ruido cardíaco (R2) (por cierre tardío de la válvula pulmonar). En algunos casos, cuando el defecto es muy grande se pueden encontrar síntomas y signos de insuficiencia cardiaca (disnea, taquicardia).

Rara vez estos pacientes están medicados, salvo en caso de defectos grandes.

Como en todos los pacientes cardiópatas es apropiado conocer la evaluación cardiológica más reciente junto con el último examen ecocardiográfico para tener una idea de la repercusión del shunt y de la función cardiaca. En todo caso, en la mayoría de los pacientes que tienen un defecto pequeño, no es necesario solicitar un examen ni una evaluación por especialista en caso de no contar con una reciente. El antecedente de la cardiopatía y el examen clínico actual bastan para programar una técnica anestésica adecuada.

Durante la anestesia el objetivo es disminuir o al menos no acentuar el shunt, por lo que las maniobras son similares al paciente con un CIV: el anestesiólo- go debe ventilar evitando aumentar el flujo pulmonar (uso de PEEP, $\mathrm{FiO}_{2}$ baja, ventilar con frecuencia y volúmenes bajos para aumentar discretamente la $\mathrm{pCO}_{2}$ ).

En un paciente con buena función cardiaca, los medicamentos y técnicas anestésicos no difieren de un paciente habitual.

Es importante que el anestesista recuerde que la administración intravenosa de burbujas puede provocar embolia paradojal en pacientes con defectos intracardiacos que están siendo sometidos a ventilación con presión positiva durante todo el ciclo respiratorio. Esto es crucial en circulación tipo "en paralelo" que se verá más adelante. La grilla de manejo hemodinámicos es similar a la de un paciente portador de una CIV.

\section{Ductus arterioso permeable}

El ductus arterioso permeable (DAP) representa la tercera anomalía cardiaca congénita más frecuente. Aunque propiamente no es una anomalía intracardiaca, causa un cuadro de cortocircuito de izquierda a derecha con sobrecarga de volumen y presión. El ductus arterioso es un elemento normal en la circulación fetal que permite el paso de sangre no oxigenada desde el VD hacia la aorta descendente y posteriormente a la placenta para oxigenarse, dado que los pulmones están colapsados y tienen una RVP muy elevada. A pesar que esta estructura tiene la función de cerrarse después de nacer a través de varios mecanismos, (siendo el principal un aumento en la oxemia), hay neonatos que permanecen con esta estructura patente. El grupo de mayor riesgo es el de los neonatos prematuros, quienes tienen una estructura muscular de la pared ductal muy inmadura impidiendo su cierre. Esto provoca que haya un shunt de izquierda a derecha a nivel ductal, con aumento del flujo, resistencia y presión pulmonar, sobrecargando finalmente las cavidades izquierdas.

Podemos distinguir básicamente un cuadro clínico en 2 poblaciones distintas; el del paciente mayor y el del prematuro.

En el niño mayor la presencia de DAP debe considerarse como una patología de menor gravedad que en general no provoca un problema en el desarrollo del paciente. De todas maneras es una condición que requiere ser corregida ya que con el tiempo puede provocar insuficiencia cardiaca e hipertensión pulmonar irreversible. En la actualidad el cierre percutáneo endovascular del defecto es el tratamiento de elección.

En pacientes prematuros el cuadro es especialmente florido y puede provocar insuficiencia cardiaca 
que requiera conexión a ventilador mecánico y uso de drogas vasoactivas para apoyar la función cardiaca. Para lograr el cierre se utiliza regularmente indometacina, ibuprofeno y recientemente paracetamol (aún en estudio).

De no ser exitoso el tratamiento médico se realiza el cierre quirúrgico del ductus. Debido a la falla cardiaca y a otros factores predisponentes, estos pacientes pueden presentar cuadros agudos abdominales (enterocolitis necrotizante) que necesiten cirugía y por lo tanto manejo anestésico por no especialista (antes de la cirugía de cierre del DAP). (Figura 4. Ductus Arterioso Persistente (DAP)).

\section{Evaluación preanestésica}

En el paciente prematuro deben evaluarse cuidadosamente la condición actual del paciente, presencia de infecciones pulmonares, requerimientos y parámetros de la ventilación mecánica (para intentar reproducirlos en el quirófano), presencia de anemia, estado de la coagulación, estado metabólico, uso de drogas vasoactivas y presencia y calidad de accesos venosos periféricos y centrales, entre otros.

\section{Anestesia}

El objetivo será mantener la mayor estabilidad hemodinámica posible, evitando acentuar el shunt y la sobrecarga de volumen, manejar apropiadamente los parámetros metabólicos (ácido base, calcemia, glicemia, entre otros). La grilla es similar a la de un paciente portador de una CIV de gran tamaño, pero haciéndose cargo de las particularidades de un paciente prematuro (riesgos de hemorragia cerebral, de hipoglicemia, escasa tolerancia a aumentos bruscos de la pre y postcarga, etc.).

Ocasionalmente un anestesiólogo general puede verse enfrentado a una cirugía de cierre de ductus ya que este procedimiento a veces puede realizarse en hospitales generales si hay cirujanos entrenados. Cabe mencionar que el acceso quirúrgico para su cierre es por toracotomía lateral. A pesar que en manos experimentadas es un procedimiento rápido, para acceder al DAP es necesario retraer el pulmón (izquierdo si el arco de la aorta es izquierdo). Sumado a la congestión y hepatización pulmonar, (hallazgo frecuente en pacientes de esta categoría, es decir prematuros en insuficiencia cardiaca), el paciente puede caer rápidamente en hipoxia profunda, tanto por alteración ventilación/perfusión como por reversión temporal

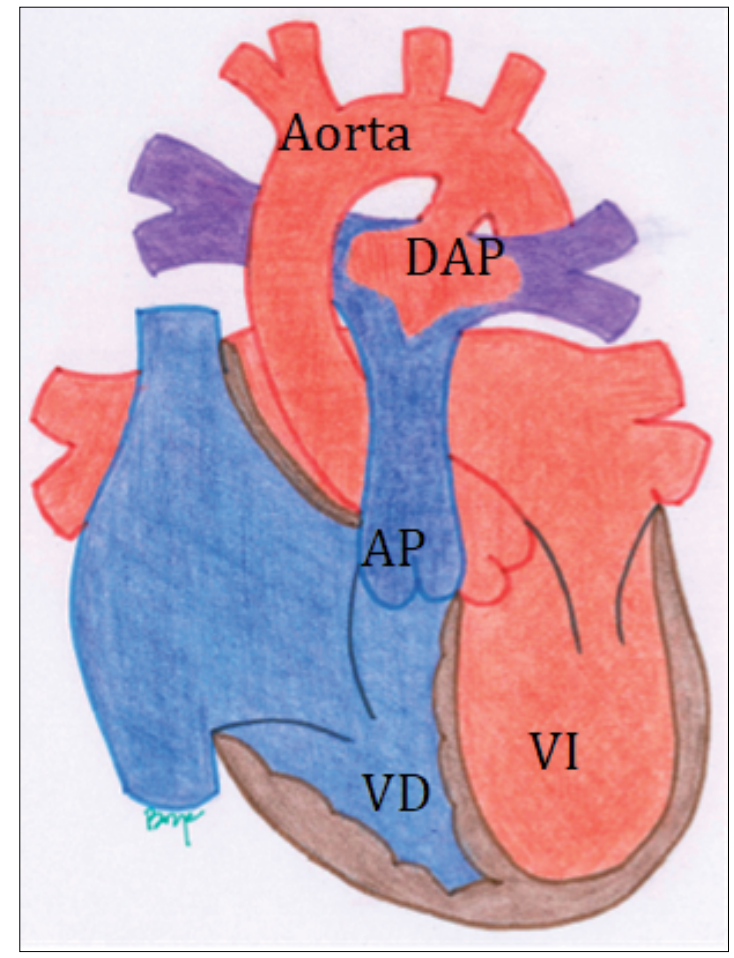

Figura 4. Ductus Arterioso Persistente (DAP). A pesar que esta estructura tiene la función de cerrarse después de nacer a través de varios mecanismos, siendo el principal un aumento en la oxemia, hay neonatos que permanecen con esta estructura permeable. El grupo de mayor riesgo es el de los neonatos prematuros, quienes tienen una estructura muscular de la pared ductal muy inmadura, impidiendo su cierre. Esto provoca que haya shunt de izquierda a derecha a nivel ductal, con aumento del flujo, resistencia y presión pulmonar, sobrecargando finalmente las cavidades izquierdas.

del shunt del DAP, provocando bradicardia y potencialmente paro cardiorrespiratorio.

\section{Tetratología de Fallot}

La Tetralogía de Fallot (TOF) es la cardiopatía congénita cianótica más común con una incidencia de 3 en 10.000 nacidos vivos, lo que representa un 6 a $11 \%$ de todas las cardiopatías (siendo la cuarta más frecuente de todas las categoría). A pesar de que se describió como una "tetralogía", estudios del desarrollo embrionario han propuesto que esta enfermedad se debe a más bien un defecto cardiaco inicial (Monología de Fallot), que consiste en la desviación anterior del septum interventricular conal. Aunque existen muchas variantes, anatómicamente es una 
anomalía que se caracteriza por los siguientes hallazgos:

- Obstrucción en el tracto de salida del Ventrículo Derecho: esta obstrucción puede ocurrir a diferentes niveles, infundíbulo del ventrículo derecho, en la zona valvular o supravalvular pulmonar.

- CIV conoventricular.

- Dextroposición de la válvula de la arteria aorta que cabalga sobre la cresta de la CIV.

- Hipertrofia del ventrículo derecho como consecuencia tanto de la obstrucción en el tracto de salida, como la eyección a presiones sistémicas (VD hacia la aorta).

\section{Fisiopatología}

Hoy en día es raro encontrarse con un paciente con una TOF no corregida, debido que ha cambiado el enfrentamiento quirúrgico de la patología. La conducta cardiológica es realizar tempranamente la corrección quirúrgica de la patología cardiaca. Aun así es relevante conocer el manejo de pacientes no corregidos. Funcionalmente se encuentra la siguiente situación:

- Flujo reducido de sangre hacia los pulmones (Qp:Qs menor de 1:1).

- Paso de sangre no oxigenada a la aorta y al territorio sistémico a través de la comunicación interventricular (cortocircuito de derecha a izquierda). El grado y severidad del shunt de derecha a izquierda depende del grado de obstrucción pulmonar pero también de otras variables como la resistencia vascular sistémica. Ante episodios de disminución de la RVS, de la pre, postcarga, ante cuadros de hipovolemia, (ayuno prolongado, episodios de diarrea ), el shunt de Derecha a Izquierda puede acentuarse a niveles peligrosos con desaturación severa. Cuando se produce esta situación clínica se dice que el paciente presenta una "crisis anoxémica".

- Cianosis variable con eritrocitosis compensatoria. El hematocrito puede ser tan alto como $50 \%$ o más. Cuando el hematocrito excede $50 \%$ es un signo inequívoco de la cronicidad y severidad de la cardiopatía. (Figura 5. Tetralogía de Fallot).

\section{Evaluación preoperatoria}

Si la patología no está corregida es relevante evaluar las condiciones clínicas generales, la saturación periférica, el nivel de hemoglobina y la medicación actual. Un hematocrito sobre $50 \%$ es una condición de riesgo y hematocritos sobre $65 \%$ pueden requerir una flebotomía antes de la anestesia para disminuir la viscosidad de la sangre. Es posible que estos pacientes estén bajo medicación con $\beta$-bloqueadores (habitualmente propanolol) si la obstrucción del tracto de salida del ventrículo derecho es dinámica. El uso de $\beta$-bloqueadores permite disminuir la obstrucción y facilita entonces el flujo hacia la arteria pulmonar. En este caso los pacientes presentaran una frecuencia cardiaca más baja.

La ecocardiografía debe ser revisada en forma acuciosa para determinar sobre todo el grado de obstrucción del tracto de salida, el nivel de la obstrucción, y si ésta es fija y/o dinámica. Si la obstrucción es fija, lo más relevante es mantener la RVS elevada con volemia adecuada y con vasoconstrictores. Si la obstrucción es dinámica (infundibular) es probable que el paciente se encuentre bajo medicación con $\beta$ bloqueadores.

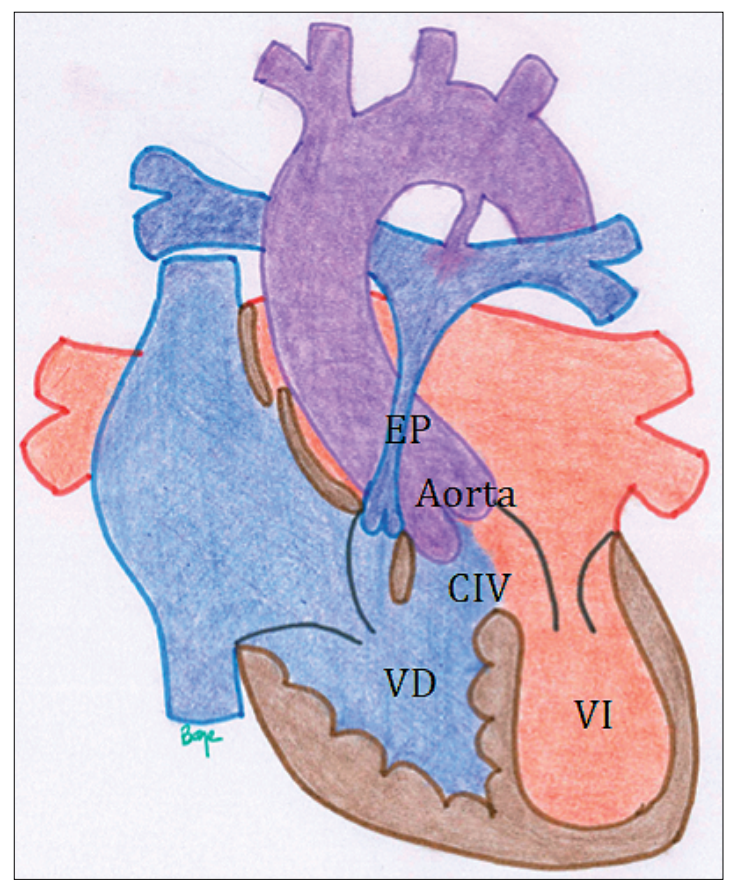

Figura 5. Tetralogía de Fallot: A pesar de su nombre, hoy en día se entiende que es una monología, en la que el defecto inicial es la desviación anterior del septum conal. Esto provoca estenosis pulmonar (EP), acompañada de comunicación interventricular (CIV) conoventricular (subaórtica), dextroposición de la Aorta (cabalgamiento) y la consecuencia en el ventrículo derecho, hipertrofia. La concomitancia de CIV y EP provoca shunt de $D^{a}$ a la en la CIV, con la resultante de paso de sangre desaturada a la circulación sistémica (cianosis). 


\begin{tabular}{|c|c|}
\hline \multicolumn{2}{|c|}{$\begin{array}{c}\text { Grilla para el manejo de la Tetralogía de Fallot no } \\
\text { corregida }\end{array}$} \\
\hline Contractilidad & $=0 \downarrow$ \\
\hline Función cardiaca & $=$ \\
\hline Precarga & $\uparrow$ \\
\hline RVP & $\uparrow$ \\
\hline RVS & $\downarrow$ \\
\hline
\end{tabular}

\section{Anestesia}

El manejo anestesiológico debe estar destinado a mantener la RVS pero también a evitar el aumento de la obstrucción en el tracto de salida del ventrículo derecho, todo ello con la intención de inducir el paso de sangre a través de la válvula pulmonar hacia los pulmones. La inducción inhalatoria solo está permitida con sevoflurano, en ningún caso con isoflurano o desflurano. La mantención debe hacerse con medicamentos que no disminuyan la RVS, es decir isoflurano y propofol están contraindicados. La inducción intravenosa con Ketamina (versus inducción por inhalación) preserva la RVS y puede evitar las crisis anoxémicas durante la inducción. También puede ser útil el uso de la Ketamina por vía intramuscular en los pacientes que están muy ansiosos y no toleran la inducción por mascarilla. Más importante que la elección del fármaco o método de inducción, es el realizar una titulación cuidadosa de los anestésicos, una preparación y planificación adecuada del procedimiento anestésico, junto con poseer los conocimientos y las habilidades para manejar una situación que es de alto riesgo anestésico. En caso de producirse una crisis anoxémica, las maniobras deben estar destinadas a aumentar la resistencia periférica, evitando al mismo tiempo aumentar la contractilidad, por lo cual los inótropos $\beta_{1}$ agonistas como la dopamina y la adrenalina no están indicados. Puede ser necesario utilizar vasopresores con efecto $\alpha_{1}$ agonistas tales como la fenilefrina, aporte de volumen generoso (dada la presencia de estenosis pulmo-

\begin{tabular}{|c|c|}
\hline \multicolumn{2}{|c|}{$\begin{array}{c}\text { Grilla para el manejo de la Tetralogía de Fallot no } \\
\text { corregida }\end{array}$} \\
\hline Contractilidad & $=$ \\
\hline Función cardiaca & $=$ \\
\hline Precarga & $\uparrow$ \\
\hline RVP & $\uparrow$ \\
\hline RVS & $\downarrow$ \\
\hline
\end{tabular}

nar, la chance de provocar edema pulmonar agudo es virtualmente inexistente). Como maniobra desesperada y transitoria, la compresión manual del abdomen con doble objetivo: primero, para comprimir la aorta abdominal aumentando la presión arterial y favorecer el flujo sanguíneo al corazón y SNC; y segundo, para comprimir el hígado y favorecer el retorno venoso, aumentando la precarga. (Tabla 6. Grilla para el manejo de la Tetralogía de Fallot).

Algunos pocos pacientes en la época actual pueden ser portadores de un Shunt Sistémico pulmonar (Shunt Blalock-Taussig Modificado [SBT]). En este caso $\mathrm{La} \mathrm{PaO}_{2}$ está determinada por una resistencia fija (el diámetro del shunt), por la gradiente de presión y el hematocrito. Para que el shunt funcione se debe mantener la presión sistémica y un hematocrito $\geq$ normal, (> 40\%). Si en un paciente portador de un SBT cae bruscamente la saturación hay que aumentar la presión arterial sistémica, revisar la ventilación y asegurarse que shunt esté aún permeable. Este último punto es vital, ya que la combinación de eritrocitosis (que eleva la viscosidad sanguínea), hipovolemia por ayunas y ventilación a presión positiva puede disminuir el flujo por la prótesis vascular, provocando trombosis de ésta. Si esta es la situación, es una emergencia vital, la que debe ser asistida con heparinización del paciente, aumentar las presiones sistémicas y pronta derivación a especialista. Nuevamente la auscultación del paciente es importante ya que dentro de la evaluación se debe auscultar el soplo continuo del SBT (Tabla 7).

\section{Referencias}

1. Jenkins KJ, Gauvreau K, Newburger JW, Spray TL, Moller JH, lezzoni LI. Consensus-based method for risk adjustment for surgery for congenital heart disease. J Thorac Cardiovasc Surg 2002 Jan; 123(1):110-8.

2. Ramamoorthy $C$, Haberkern
CM, Bhananker SM, Domino KB, Posner KL, Campos JS et al. Anesthesia-related cardiac arrest in children with heart disease: data from the Pediatric Perio- 
perative Cardiac Arrest (POCA) registry. Anesth Analg 2010 May;110(5):1376-82.

3. White MC, Peyton JM Anaesthetic management of children with congenital heart disease for non-cardiac surgery. Continuing Education in Anaesthesia. Critical Care \& Pain J 2012;12:17-22.

4. Baum VC, Palmisano BW. The immature heart and anesthesia. Anesthesiology 1997 Dec;87(6):1529-48.

5. Allen U. Infective endocarditis: updated guidelines. Can J Infect Dis Med Microbiol 2010;21(2):74-7.

6. Anesthetic agents and their cardiovascular effects. Chapter 6 . In Anesthesia for Congenital Heart Disease. Andropoulos DB. $3^{\mathrm{a}} \mathrm{Ed}$.
2015. Wiley.

7. Friesen $\mathrm{RH}$. Anesthetic drugs in congenital heart disease. Semin Cardiothorac Vasc Anesth 2014 Dec;18(4):363-70.

8. Gottlieb EA, Andropoulos DB. Anesthesia for the patient with congenital heart disease presenting for noncardiac surgery. Curr Opin Anaesthesiol 2013 Jun;26(3):318-26. 